\title{
ESTUDIO DE IMPACTO AMBIENTAL DEL PROYECTO DE RELLENO SANITARIO EN LA COMUNIDAD DE MORALILLO, PROPUESTO PARA LA CIUDAD DE IQUITOS, LORETO-PERÚ
}

\author{
Melba Correa T. ${ }^{1}$, Bertha Montalván I. ${ }^{2}$, Roberto Pezo D. ${ }^{3}$, Lorgio Verdi O. ${ }^{3}$
}

\section{RESUMEN}

El presente estudio se realizó en la Comunidad de Moralillo, Distrito de San Juan Bautista de la ciudad de Iquitos, durante los meses de octubre 2005 a marzo 2006, para evaluar el impacto ambiental que ocasionaría la construcción de un Relleno Sanitario en la mencionada comunidad. Se realizó una descripción de la zona de estudio, en un área de 49,753.93 m2, referida a aspectos físicos, biológicos y socioeconómicos y a las actividades del proyecto; ambas informaciones fueron procesadas en la matriz de Leopold, para determinar los impactos que ocasionaría el establecimiento de un relleno sanitario en Moralillo.

Los resultados demuestran que el área destinada no es la adecuada por razones biofísicas y legales, por no cumplir con algunos requisitos básicos para la implementación de este tipo de actividad. Además en caso se persista con la idea de implementar el mencionado relleno en la comunidad de Moralillo, se presenta una propuesta de Plan de Manejo Ambiental, donde se incluye algunas medidas de mitigación y compensación ambiental, para minimizar los daños al entorno y a la población.

PALABRAS CLAVE: Relleno sanitario, impacto ambiental, medio ambiente, Moralillo.

\section{ENVIRONMENTAL IMPACT STUDY FOR THE LANDFILL PROJECT IN THE MORALILLO COMMUNITY, PROPOSED FOR IQUITOS CITY, LORETO - PERU}

\begin{abstract}
The study was carried on at Moralillo Community, San Juan District, Iquitos city, between October 2005 and March 2006, to evaluate the environmental impact of a landfill facility will occasionate on the Community. A description of the study area was made, a total of $49,753.93 \mathrm{~m} 2$, referred to its physical- biological, socio- economical aspects and activities from the project, both of them were processed on the Leopold matrix, to determinate the impacts that may set with the construction of a landfill at Moralillo.

The results show that the area intended for the construction of the facility is not the appropriate one, due to biophysical and legal grounds, it does not full fill the basic requirements for the implementation of this kind of activity. Besides, in the case to persist with the implementation in the area, a proposal of an Environmental Management Plan is presented, with some mitigation and compensation measures to minimize the damage to the environment and the population.
\end{abstract}

KEYWORDS: Landfill, environmental impact, environment, Moralillo.

1 Coordinadora Educación Ambiental del Instituto de Investigaciones de la Amazonía Peruana. rcorrea@iliap.org.pe

2 Docente Asociada, Facultad de Medicina Humana de la Universidad Nacional de la Amazonía Peruana. marisol_0107@hotmail.com

3 Docente Principal, Facultad de Ciencias Biológicas de la Universidad Nacional de la Amazonía Peruana. 


\section{INTRODUCCIÓN}

En el mundo, en los últimos años se ha venido registrando una creciente preocupación por el estado actual y futuro del medio ambiente, debido a los daños ocasionados por la contaminación producto de los residuos sólidos, aunque se ha hecho muy poco para solucionar este problema, planteándose como alternativa, la creación de los denominados rellenos sanitarios, que se constituye en una técnica para la disposición de residuos sólidos en el suelo sin causar perjuicio al medio ambiente y sin causar molestias o peligro para la salud y seguridad pública (Díaz 2001, Palacios et al. 2000, ASCE 2000, Odriozola 2004). En Iquitos la gestión de los residuos sólidos está a cargo de la Municipalidad Provincial de Maynas, que se limita a la recolección domiciliaria no clasificada y su depósito en un botadero a cielo abierto ubicado en el $\mathrm{km} 1.5$ de la carretera Iquitos-Nauta. El inadecuado tratamiento de los residuos sólidos está propiciando problemas ambientales y de salud, por la proliferación de organismos patógenos, malos olores, presencia de roedores y moscas (vectores de enfermedades), y el indiscriminado aumento de "gallinazos" Coragyps atratus, perjudicando seriamente el transporte aéreo en la ciudad, por la cercanía del botadero con el aeropuerto internacional de Iquitos. En este sentido, el presente estudio tiene por finalidad evaluar el impacto ambiental que ocasionaría la propuesta de relleno sanitario en la comunidad de Moralillo, sirviendo como herramienta en la toma de decisiones por parte de las autoridades pertinentes, a fin de poder establecer un equilibrio entre el desarrollo de la actividad humana y el medio ambiente, mejorando nuestro entorno y calidad de vida.

\section{MATERIALES Y MÉTODOS}

\section{ÁREADEESTUDIO}

Ubicado a la altura del $\mathrm{km} 15.5$ de la carretera Iquitos-Nauta, a $4.5 \mathrm{~km}$ con dirección sur este, por una carretera de penetración hacia la Comunidad de Moralillo, Distrito de San Juan, Provincia de Maynas, Departamento de Loreto (coordenadas UTM 684774 E $9566069 \mathrm{~N}$ ), con un área total de $49,753.93 \mathrm{~m}^{2}$.

\section{AMBIENTEFÍ́SICO}

Se realizaron análisis físico-químicos y bacteriológicos de las aguas superficiales, estableciendo tres puntos de muestreo representativos. Así mismo, se tomaron muestras de suelo de la zona de estudio, que luego fueron analizadas en el laboratorio de la Universidad Agraria La Molina, para posteriormente describirlos de acuerdo a normas y criterios establecidos.

Ambiente Biológico. Se establecieron unidades de muestreo de vegetación, ubicados de manera que representaran todos los tipos de vegetación presentes, entre ellas dos parcelas de $10 \times 100 \mathrm{~m}$, uno en una "purma" y otro en un bosque tipo "aguajal"; una parcela de $20 \times 50 \mathrm{~m}$ en un bosque poco intervenido de tierra firme; y tres parcelas de $10 \times 100 \mathrm{~m}$ en el área que corresponde al relleno sanitario propuesto. En cada unidad se colectaron muestras botánicas que fueron identificadas en el Herbarium Amazonense de la Universidad Nacional de la Amazonía Peruana. Así mismo, para evaluar la fauna, se establecieron cinco transectos de $200 \mathrm{~m}$ de longitud, donde se realizaron censos diurnos y nocturnos, empleando el método directo, que consistió en reconocer al animal de manera visual $\mathrm{o}$ auditiva; y el método indirecto, que consistió en reconocer indicios de animales.

\section{AMBIENTE SOCIAL Y ECONÓMICO}

Se realizaron encuestas a los pobladores de Moralillo y Varillal, recogiendo información acerca de salud, unidad familiar, migración, aspectos culturales, demografia, infraestructura de servicios, actividades económicas, usos de suelo, y las perspectivas de la población respecto a la construcción del relleno sanitario.

Evaluación del impacto ambiental y Propuesta de Plan de Manejo. Para relacionar la causa y el efecto entre las actividades del relleno sanitario y los impactos en el ambiente se utilizó la matriz de Leopold et al.1971, calificando la magnitud del impacto en una escala del 1 al 3, siendo 3 mayor impacto, así como el signo $(+)$ si el efecto es positivo y un signo (-) si el efecto es negativo. Para complementar el estudio, y en caso se ejecutará la construcción del relleno sanitario en la comunidad de Moralillo, se propone un Plan de Manejo Ambiental, señalando procedimientos básicos a seguir, que procuran minimizar los impactos negativos de las actividades de construcción del relleno sanitario.

\section{EVALUACIÓN DEL IMPACTO AMBIENTALY PROPUESTA DEPLAN DEMANEJO}

Para relacionar la causa y el efecto entre las actividades del relleno sanitario y los impactos en el ambiente se utilizó la matriz de Leopold et al.1971, calificando la magnitud del impacto en una escala del 1 al 3 , siendo 3 mayor impacto, así como el signo (+) si el efecto es positivo y un signo (-) si el efecto es negativo. Para complementar el estudio, y en caso se ejecutará la construcción del relleno sanitario en la comunidad de Moralillo, se propone un Plan de Manejo Ambiental, señalando procedimientos básicos a seguir, que procuran minimizar los impactos negativos de las actividades de construcción del relleno sanitario. 


\section{RESULTADOS}

\section{AMBIENTEFÍSICO}

Aguas Superficiales. Existen "humedales" y aguajales, cuyas aguas toman el curso de las "quebradas" de la zona, que posteriormente desembocan en el río Itaya. Estos ambientes acuáticos son oligotróficos, de baja productividad, ligeramente alterados por actividades humanas, y que por su carácter somero son muy sensibles a los cambios ambientales. Los parámetros físico-químicos $\mathrm{y}$ bacteriológicos están dentro de los límites permisibles para este tipo de cuerpos de agua.

Suelos. Se presentan dos tipos de suelos: suelos de origen aluvial reciente, compuesto por arena, limo y arcilla, situados en los valles intercolinosos, adyacentes al "humedal" y a las "quebradas" involucradas en el área de estudio, que reciben continuamente sedimentos de ellos, con pendientes planas a ligeramente inclinadas $(0-2 \%)$, mal drenadas y de baja fertilidad, aptos como tierras de protección con limitaciones de drenaje con un grado de inundabilidad ligero a moderado; y suelos aluviales antiguos, con pendientes que oscilan entre $25-40 \%$, con drenaje interno bueno y una rápida escorrentía superficial, la textura es moderadamente gruesa a moderadamente fina, con una fertilidad natural baja, con aptitud potencial para forestales por la pendiente empinada y con limitaciones debido a la fertilidad baja y susceptibilidad a la erosión hídrica.

\section{AMBIENTE BIOLÓGICO}

Vegetación. El área de estudio presenta: bosques tipo terraza, un bosque tipo aguajal mixto, bosques secundarios tipo "purmas" y "chacras", y un bosque secundario completamente abierto ubicado en el área designada para construir el relleno sanitario. Se pueden apreciar especies útiles entre maderables, para construcción, comestibles, medicinales, forrajeras y ornamentales. Los bosques secundarios tipo "purmas" y "chacras" son predominantes en la zona, y se encuentran diseminados por toda el área de influencia que corresponde al relleno sanitario.

Fauna. La zona de estudio, por ser intervenida, no presenta abundancia y diversidad de especies animales, observándose solamente aves y mamíferos menores, indicadores de hábitats intervenidos, siendo las especies de aves más abundantes Brotogeris versicolorus, Aratinga weddelli, Crotophaga ani, Crotophaga major, Thraupis episcopus, Thraupis palmarum y Ramphocellus carbo, y entre los mamíferos, solo fue posible visualizar la especie Saguinus fuscicollis (Primate), puesto que la presencia de otras especies de mamíferos se registraron solo por indicios (huellas, despojos, entrevistas a moradores).

\section{AMBIENTESOCIAL}

Población. La población del área de influencia se estima en 3,189 habitantes, de los cuales, 407 personas $(55,9 \%$ varones y $44,1 \%$ mujeres) pertenecen a la comunidad de Moralillo, y 2,782 (54,1\% varones y $45 \%$ mujeres) a Varillal, observándose un crecimiento poblacional muy acelerado según referencias de años anteriores, existiendo mucha migración de los más jóvenes a la ciudad de Iquitos por motivos de estudio y trabajo.

Salud. La frecuencia de enfermedades es baja, siendo los únicos problemas los casos de malaria y enfermedades diarreicas y respiratorias, las que son atendidas en la posta médica.

Vivienda. Son de tipo urbano y rural, y la combinación de ambos tipos. La mayoría cuenta con los ambientes básicos necesarios y la mitad no presentan título de propiedad.

Comunicación. Varillal cuenta con servicios de transporte que los conecta con las ciudades de Nauta e Iquitos, así como radio y telefonía, lo contrario sucede en Moralillo, que solo dispone de transporte restringido y donde los servicios de radio y telefonía no existen.

Organización. Ambas comunidades cuentan con autoridades elegidas en asambleas comunales como el Teniente Gobernador y el Agente Municipal. No disponen con destacamento de la Policía Nacional, pero sí tienen apoyo de la policía de carreteras.

Percepción de la población sobre la construcción del relleno. La mayoría de los pobladores de Varillal $(76,79 \%)$ y Moralillo (84\%) muestran conformidad con la construcción del relleno sanitario, pues perciben que se crearán fuentes de trabajo que los beneficiará, sin embargo, también consideran que se pueden generar problemas ambientales.

\section{AMBIENTEECONÓMICO}

La economía local se sustenta en tres actividades principales: la actividad económica productiva; orientada principalmente al cultivo de especies de "pan llevar" y "caña de azúcar", la actividad pecuaria y cultivo de peces; la actividad económica de transformación, dirigida a la elaboración de aguardiente de manera artesanal; y la actividad económica de servicios, basada en guardianías en chacras y piscigranjas y como trabajadores en la elaboración de aguardiente; además, un grupo importante de pobladores realiza actividades comerciales, a través del expendio de productos de primera necesidad. A pesar de esto, en ambas comunidades existe un significativo porcentaje de desempleo.

Impactos Ambientales del Relleno Sanitario y Plan 


\section{de Manejo}

Según la matriz de Leopold se identificaron los siguientes posibles impactos:

\section{AMBIENTE FÍsICO}

Suelo y Aguas Superficiales. En la fase de construcción, el suelo sufrirá mayor impacto a causa de la deforestación para "despejar" el terreno donde se construirá el relleno sanitario. El impacto sería adverso, mitigable, local, permanente, significativo, irreversible en los caminos de acceso y reversible en el área del relleno sanitario. En la fase de funcionamiento, el suelo y las aguas superficiales podrían sufrir un impacto de tipo adverso, mitigable, local, permanente e irreversible, a causa de los lixiviados. En la fase de abandono, el suelo se vería beneficiado significativamente por su fertilización a causa de la descomposición de la materia orgánica, y las aguas superficiales dejarían de recibir descargas de los lixiviados, recuperándose para poder ser útiles. Como medidas de mitigación, los suelos deberían ser impermeabilizados con material adecuado y duradero, y se sembrarían cortinas vegetales alrededor del relleno. Como medidas compensatorias, se debería mejorar la calidad del puesto de salud de la Comunidad de Moralillo.

\section{AMBIENTE BIOLÓGICO}

Vegetación y Fauna. En la fase de construcción, la vegetación y la fauna sufrirían un impacto de tipo medio-alto, adverso, no mitigable, local, permanente, no significativo e irreversible a causa de la deforestación, desplazando a la escasa fauna del lugar por eliminación de sus hábitats. Así mismo, el paisaje sufriría un impacto de tipo adverso, bajo, no mitigable, local, permanente, no significativo e irreversible, por alteración de la belleza escénica de la zona. Además, la emisión de ruidos, se considera impacto de tipo bajo a medio, adverso, mitigable, local, temporal, no significativo y reversible. En la fase de funcionamiento, los lixiviados producirían mayor impacto, de tipo adverso, mitigable, local, permanente e irreversible, pues podrían llegar a los cuerpos de agua y diseminarse por los aguajales de la zona y la vegetación adyacente. Además, los ruidos producirían un impacto adverso en la fauna del lugar, obligando a los animales a desplazarse a lugares más alejados, este impacto es de tipo mitigable, local, permanente e irreversible. En la fase de abandono, la vegetación y la fauna se verían beneficiados significativamente por la recuperación de los bosques por regeneración y reforestación. Como medidas de mitigación, el tratamiento de la basura se debería realizar con equipos modernos minimizando los riesgos de contaminación. Como medidas compensatorias, se podría mejorar la infraestructura educativa $y$ desarrollar campañas de educación ambiental que sensibilicen y capaciten a los pobladores locales en el adecuado manejo de los desechos.

\section{AMBIENTESOCIO-ECONÓMICO}

En la fase de construcción se podría presentar en la población un impacto de tipo medio-alto, beneficioso, local, temporal y significativo, por los ingresos económicos producto del empleo en las labores de construcción. En la fase de funcionamiento, las fuentes de empleo creadas en forma directa e indirecta, también podrían representar un impacto beneficioso de tipo medio-alto, local, temporal y significativo. Así mismo, las vías de acceso construidas hacia la comunidad de Moralillo, beneficiarían a todas aquellas personas que tengan que sacar sus productos agrícolas hacia la ciudad. Por otro lado, y debido a la proliferación de fauna nociva (moscas, ratas, gallinazos, etc.), se puede presentar cierto cambio desfavorable en el estilo de vida de los pobladores. En la fase de abandono, la población se vería altamente beneficiada por el empleo en las actividades de reforestación y retiro de las instalaciones del relleno sanitario. Las medidas de mitigación refieren un control de plagas adecuado; así mismo, los puestos de trabajo creados en forma directa e indirecta deberán ser desarrollados por los pobladores locales. Como medidas compensatorias, sería conveniente implementar con los servicios básicos a las comunidades implicadas, brindándoles servicio de alumbrado eléctrico, agua potable y servicio de comunicaciones.

\section{DISCUSIÓN}

El área destinada para la construcción del relleno sanitario en la Comunidad de Moralillo, no es la más adecuada, resultados que se presentan opuesto a lo afirmado por la Municipalidad Provincial de Maynas (2005), debido a que no cuenta con algunas características tanto físicas, biológicas y socioeconómicas básicas para su desarrollo en esta área, contraviniendo a los criterios que se encuentran en el Art. $67^{\circ}$ Criterios para la selección de áreas de infraestructuras de residuos sólidos, Reglamento de la Ley 27314, Ley General de Residuos Sólidos, Título V, Capítulo I (2004), por lo que su construcción podría resultar perjudicial al medio ambiente y como consecuencia a los pobladores de la zona, puesto que como lo señalan el Banco Interamericano de Desarrollo (1997) y Roel \& Sánchez (2004), la mala ubicación de un relleno sanitario podría generar contaminación ambiental e impactos a la estética, a la salud pública y ocupacional, alterando el medio ambiente natural en forma negativa y causando impactos adversos en la población circundante, puesto 
que el desarrollo de este tipo de proyectos toma en cuenta algunas recomendaciones acerca de su ubicación e implementación para que los impactos que genere puedan ser mitigados (Consejo Consultivo De La Comisión Regional Del Medio Ambiente, Región De La Araucaria 2003), toda vez que implica acciones impactantes como eliminación de cubierta vegetal y movimiento de tierras que producen ruidos molestos y alteran la hidrología (Roel \& Sánchez 2004); así mismo, su funcionamiento produce impactos en la atmósfera, al suelo y cuerpos de agua tanto superficiales como subterráneas, entre otros impactos (Mujica \& Mesa 1997).

En el ambiente físico, el área estudiada presenta cuerpos de agua superficiales, característica que no lo hace apropiado para implementar un relleno sanitario, pues se recomienda que éstos deben realizarse en lugares alejados de cuerpos de agua (IIAP 2005, United Nations Environment Programme Division Of Technology, Industry And Economics 2004), tal como lo señala Elgueta (2005), serían muy susceptibles de ser contaminadas, comprometiendo otros sistemas ecológicos, como los bosques adyacentes e interiores hacia el río Itaya. Por otro lado, el suelo se vería afectado en la fase de construcción por la deforestación realizada para "despejar" el terreno, aumentando, como también lo indica García et al. (2004) los riesgos de erosión del suelo. Además, un relleno sanitario debe ubicarse en terrenos con pendientes que no superen el $20 \%$ (IIAP 2005), ya que sumado a las precipitaciones muy continuas, puede contribuir a la diseminación de los lixiviados; de igual manera, los suelos deben presentar un alto índice de impermeabilidad (Elgueta 2005), lo que no ocurren con la ubicación del actual proyecto de relleno sanitario en Moralillo, corriendo el riesgo de que los lixiviados generados contaminen los acuíferos y cuerpos de agua superficiales (Damonte 2004) y por consiguiente, a las personas que consumen estas aguas, tal como lo señala Odriozola (2004), los lixiviados contienen altos niveles de metales tóxicos y compuestos orgánicos capaces de producir cáncer. En la fase de abandono, y siempre que se haya realizado un adecuado trabajo de reciclaje de los desechos, el suelo podría ser beneficiado por su fertilización, aumentando su calidad (García et al. 2004, Ingeniería Ambiental \& Medio Ambiente 2000), de lo contrario, estos suelos perderán su valor.

En cuanto al ambiente biológico, la zona de Moralillo, presenta principalmente bosques secundarios, con algunos sectores de bosque poco intervenido, por lo que en la fase de construcción, la vegetación y la fauna sufrirían un impacto adverso por la deforestación (García et al. 2004), pues se eliminarían los hábitats de la fauna que todavía queda en el lugar, alterando además el paisaje, que sumado a la contaminación sonora (Roel \& Sánchez 2004), ocasionarían el desplazamiento de los animales hacia lugares más alejados. En la fase de funcionamiento, los lixiviados podrían producir mayor impacto, pudiendo contaminar la vegetación del lugar por su diseminación por los cuerpos de agua (Damonte 2004), afectando a los animales que utilizan estos bosques como refugio, en esta fase, también la fauna se vería afectada por las emisiones de ruidos molestos que las alejarían de la zona (Roel \& Sánchez 2004). En la fase de abandono, la vegetación y la fauna serían beneficiados por actividades de reforestación García et al. (2004), que sumado a la regeneración natural, recuperaría los bosques impidiendo además la erosión de los suelos.

La economía local se sustenta en actividades que solo captan a la mitad de la población, por lo que existe mucho desempleo, y que sumado al acelerado crecimiento demográfico (Wahl et al. 2003), propicia la migración hacia la ciudad en busca de trabajo. En este sentido, los pobladores muestran conformidad con la construcción del relleno sanitario por la creación de empleos que los beneficiará, aunque consideran, que se pueden generar problemas ambientales, principalmente por la pérdida del valor de las tierras agrícolas (García et al. 2004), puesto que los terrenos podrían perder su potencial en actividades productivas (Damonte 2004). Así mismo, el centro poblado se encuentra muy cercano al lugar donde se plantea establecer el relleno sanitario, a pesar de que se recomienda que este tipo de proyectos deben realizarse alejados de centros poblados a distancias no menor de $5 \mathrm{~km}$ (IIAP 2005, Allende 2001, United Nations Environment Programme Division Of Technology, Industry And Economics 2004), situación que en la fase de funcionamiento, podría ocasionar problemas de salud graves en la población, pues existen riesgos asociados a la salud mental debido al deterioro anímico (Ingeniería Ambiental \& Medio Ambiente 2000), riesgos de contraer algún tipo de cáncer, así como disminución de peso y talla en los infantes (Odriozola 2004), toda vez y como lo señalan Hollie \& McRae (1997), muchos residuos contienen elementos tóxicos, que serían liberados en los gases, polvos, acompañado de malos olores y humos (Mujica \& Mesa 1997, Damonte 2004), que serían dispersados por los vientos a los centros poblados. La descomposición de la materia orgánica generaría microorganismos patógenos que podrían producir enfermedades transmitidas por vectores (Roel \& Sánchez 2004), como ratas, cucarachas, moscas, mosquitos, que además ocasionaría un cambio de vida en la población al proliferar este tipo de fauna nociva (Mujica \& Mesa 1997), sin dejar de mencionar la 
proliferación de los "gallinazos" Coragyps atratus que causan serios problemas en el transporte aéreo a la ciudad de Iquitos.

En caso se establezca el relleno sanitario en Moralillo, se deberá elaborar un adecuado diseño del relleno y usar equipos modernos que permitan minimizar los impactos, como adquirir geomembranas que impermeabilicen el relleno, mitigando la percolación del lixiviado hasta las aguas subterráneas, la incorporación de sistemas de captación de lixiviados, la habilitación de ductos para la evacuación de los gases de vertedero (Roel \& Sánchez (2004), así como la implementación de cortinas vegetales que protejan a los pobladores cercanos de los hedores, polvo y emanación de gases tóxicos (Mujica \& Mesa 1997, García et al. 2004). Para complementar el tratamiento de la basura, autores como Mujica \& Mesa (1997), United Nations Environment Programme Division Of Technology, Industry And Economics (2004), refieren que la selección y el reciclaje de los desperdicios orgánicos e inorgánicos ayudaría en gran parte a solucionar los problemas de contaminación por los desechos, mediante programas que promuevan esta actividad (Roel \& Sánchez 2004), puesto que como lo señala CEAMSE (2004), en los rellenos sanitarios no se debería admitir residuos riesgosos, capaces de producir daños graves a la salud.

\section{CONCLUSIONES}

La zona de Moralillo NO ES APTA para la construcción de un relleno sanitario, por presentar una topografia muy accidentada propensa a las inundaciones por escorrentía, cuerpos de agua superficiales muy cercanos sujetos a contaminación, suelo permeable que tienden a lixiviar o percolar líquidos contaminantes hacia las napas freáticas, y centros poblados muy cercanos que pueden ser perjudicados en su salud, contraviniendo a los criterios que se encuentran implícitos en el Art. $67^{\circ}$ Criterios para la selección de áreas de infraestructuras de residuos sólidos, Reglamento de la Ley 27314, Ley General de Residuos Sólidos, Título V, Capítulo I (2004).

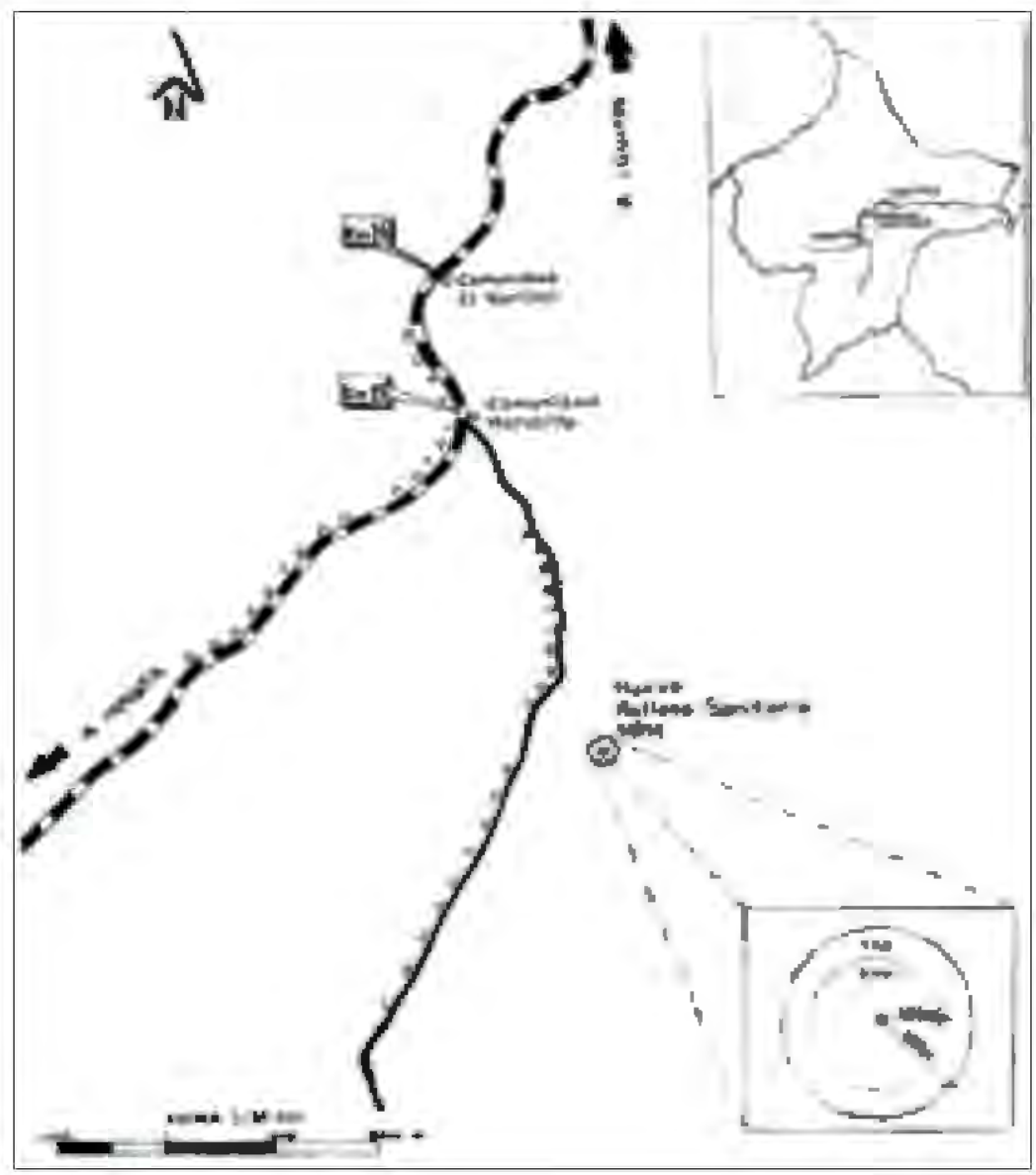

Figura 1. Mapa de ubicación del área de estudio. 
Los pobladores de las comunidades involucradas, están de acuerdo con la construcción del relleno sanitario en Moralillo, porque consideran que se crearán fuentes de empleo que los beneficiará, pero también consideran que se podría generar problemas ambientales.

Los beneficios económicos que originaría el funcionamiento del relleno en Moralillo, no compensarían los daños al medio ambiente y a la salud de los pobladores locales.

En caso de establecerse el relleno sanitario en Moralillo, el diseño del relleno deberá ser el más adecuado con equipos modernos, complementándose con tareas de selección y reciclaje de los desperdicios mediante programas que promuevan esta actividad.

\section{REFERENCIAS BIBLIOGRÁFICAS}

ALLENDE, T. 2001. Evaluación GeológicoAmbiental en la Determinación de la Factibilidad de un Área Para Relleno Sanitario. Revista del Instituto de Investigación de la Facultad de Ingeniería Geológica, Minera, Metalúrgica y Geográfica. Vol.4 no.7 Lima.

AMERICAN SOCIETY OF CIVIL ENGINEERSASCE. 2000. Sistema de relleno sanitario en el Área Metropolitana de Buenos Aires. Argentina. $11 \mathrm{pp}$.

BANCO INTERAMERICANO DE DESARROLLO. 1997. Guía para Evaluación de Impacto Ambiental para Proyectos de Residuos Sólidos Municipales.

CONSEJO CONSULTIVO DE LA COMISIÓN REGIONAL DEL MEDIO AMBIENTE, REGIÓN DE LAARAUCARIA. 2003. Propuesta para un mejoramiento en el manejo de residuos sólidos urbanos en la región de la Araucania. Temuco.12 pp.

COORDINACIÓN ECOLÓGICA ÁREA METROPOLITANA SOCIEDAD DEL ESTADO. CEAMSE. 2004. Rellenos Sanitarios. Argentina. $11 \mathrm{pp}$.

DAMONTE, G. 2004. Proyecto Integral de Valorización y Disposición Final de Residuos Sólidos Urbanos. Proyecto "A pesar de todo". Argentina. 5 pp.

DIAZ, D. 2001. Basura: Destino incierto en Venezuela. Los Rellenos Sanitarios.

ELGUETA, A. 2005. Construcción de Rellenos Sanitaros Intercomunales. La Araucania. GARCÍA, I.; M. BLANDÓN; W. VÁSQUEZ \& G. RODRÍGUEZ. 2004. Evaluación de Impacto Ambiental del Relleno Sanitario de la Ciudad de Condega. Universidad Nacional Autónoma de
Nicaragua. $65 \mathrm{pp}$.

HOLLIE N. \& G. MCRAE. 1997. 11 Recomendaciones para mejorar el manejo de los Residuos Hospitalarios. $2^{\mathrm{a}}$ edición. CGH Environmental Strategies. USA. $11 \mathrm{pp}$.

IIAP. 2005. Recomendaciones para el establecimiento del relleno sanitario para Iquitos

INGENIERÍA AMBIENTAL \& MEDIO AMBIENTE. 2000. Residuos Sólidos. http://www.fortunecity.es/expertos/profesor/171/ residuos.html. $36 \mathrm{pp}$.

LEOPOLD, L.; F. CLARKE; B. HANSHAW \& J. BALSLEY. 1971. A procedure for evaluating environmental impact. Geological Survey. Circ. 645, G.O.P., Washington DC.

MUJCA, P. \& G. MESA. 1997. Impacto Ambiental del Tratamiento de Residuos Sólidos en Mocoretá. http://www.ilustrados.com/publicaciones/EEEyy AAEZFzqmCCYNJ.

ODRIOZOLA, V. 2004. Plan de Basura Cero para Buenos Aires. Green peace. Argentina.

PALACIOS, F.; E. GARCÍA; O. GARCÍA; V. FRAGUELA; A. RUÍZ; M. SARDUY \& F. POTRILLÉ. 2000. Manejo De Residuos Sólidos Urbanos En La Zona Litoral De La Bahía D e La Habana. XIX Forum de Ciencia y Técnica del MITRANS. Cuba. 7 pp.

ROEL M. \& C. SÁNCHEZ. 2004. Villa Pehuenia, Proyecto de relleno sanitario. Facultad de Ciencias Fisicomatemáticas e Ingeniería de la Universidad Católica Argentina. $23 \mathrm{pp}$.

UNITED NATIONS ENVIRONMENT PROGRAMME DIVISION OF TECHNOLOGY, INDUSTRY AND ECONOMICS. 2004. Waste Management Planning An Environmentally Sound Approach for Sustainable Urban Waste Management - An Introductory Guide for Decision-makers. United Nations Publication. First edition. Integrative Management Series No.6.39pp.

WAHL L.; L. LIMACHI \& J. BARLETTI. 2003. Del discurso oficial al caserío rural: el desarrollo regional y la carretera Iquitos-Nauta. P.155. En Amazonía Procesos Demográficos y Ambientales. 